\section{SCAN, PLAN, FINISH, FIT}

When you buy your CEREC System from Ceramic Systems you not only get the best price and exclusive special offers, but you also get access to the best CEREC after-sales support in the UK.

CEREC enables dentists to create high quality and durable chairside all-ceramic restorations in the most cost effective and efficient way. Saving laboratory costs it enables dentists to design and create allceramic inlays, onlays, partial crowns, veneers and crowns for the anterior, premolar and molar regions in one visit. Eliminating the need for impressions, CEREC uses a digital impression taking technique to capture the data used to design the restoration which is then milled in the milling unit.

Ceramic Systems are participating in a series of One Day Scan, Plan, Finish \& Fit Digital Implementation Workshops at Sirona's new Centre of Excellence in London, on 15 June, 14 September and 7 December. Delegates will have the opportunity to hear Dr V. J. Vadgama, a preeminent user of digital equipment, explain how he has implemented a workflow that has allowed him to offer outstanding levels of patient treatment.

Numbers are limited so to book your place call Ceramic Systems Limited on 01932 582930, e-mail j.colville@ceramicsystems.co.uk or visit www.ceramicsystems.co.uk.

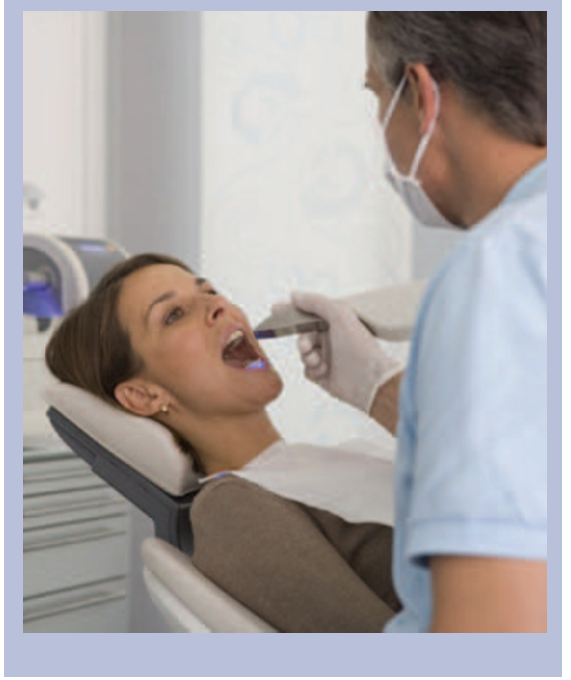

\section{EASY ACCESS TO A WEALTH OF RESOURCES}

Dentalcare.com is Oral-B and Fixodent's biggest online resource, serving dental professionals for over 15 years in different parts of the world. On average, more than 50,000 dental professionals use this website every month.

After extensive redesign and redevelopment Oral-B is now launching Dentalcare.com in the UK with UK-specific material. The new website has been designed to provide intuitive, easy access to a wealth of resources relevant to every aspect of UK dentistry.

Dentalcare.com is a complete online platform where dental professionals can find all the resources they need to help

\section{NEAT FLOWABLE RESTORATIVE}

GC Europe has launched two superior flowable materials: G-ænial Universal Flo and G-ænial Flo, to give patients the best aesthetic, functional and durable restorations.

Both flowable materials benefit from a composition that features a unique filler technology. Unlike other flowable composites the GC materials have a higher filler load and a homogeneous dispersion of fillers. The resulting improved strength and wear resistance are two key features of these flowable materials, opening up the potential for a broader use than standard flowables.

G-ænial Universal Flo is radiopaque and features a high viscosity, making it ideal for placement in Class I-V restorations. Essentially, it looks like a flowable but behaves them in every practice. All in one place, dental professionals can keep up to date with the very latest UK industry news and events, Oral-B product innovations and launches, and order both Oral-B and Fixodent samples. Access to Oral-B's continuous professional development courses together with interactive educational webinars is provided alongside high quality, comprehensive patient education, including videos on a huge range of oral health topics and leaflets to download. Together with tips and advice on working in practice and a database of over 600 research articles at your fingertips, Dentalcare.com is the one stop shop for every informed dental professional team. like a restorative. Its indications are for direct restorations, minimum intervention cavities and fissure sealing. With a physical performance of a regular composite, this material is highly thixotropic and stays neatly in place holding its shape for ease of use. It is virtually self-polishing with superb invisible aesthetics.

These restoratives are dispensed through an ergonomically designed syringe that enables smooth delivery of the material, with a tapered tip that means no paste can stick to it. The syringe size provides a comfortable hold and the arched shape of the flange fits the hand ergonomically. The design also prevents material waste because minimal residual paste remains in the syringe after use.

For further information contact GC UK on 01908218999.

\section{JUST ONE POWERFUL UNIT}

Save valuable examination time and increase confidence in the accuracy of your diagnoses with the CS 9300 Kodak Extra Oral Imaging System from Carestream Dental.

Combining medium-field cone beam CT technology with dedicated panoramic imaging, the system gives you truly adaptable 2D and 3D imaging.

Practitioners can simply select a programme and the system automatically adjusts volume size, resolution and position, scanning only the region of interest to limit radiation dose.

The CS 9300 provides an outstanding level of detail with the highest image quality in the industry. Practitioners can even generate anatomically correct images with 1:1 measurements for a precision diagnosis.

For more information, contact Carestream Dental on 08001699692 or visit www.carestreamdental.co.uk. 